\title{
PEGIDA, politische Gelegenheitsstrukturen und der neue Autoritarismus ${ }^{1}$
}

\author{
Oliver Nachtwey
}

\section{Zusammenfassung}

PEGIDA wird in diesem Beitrag nicht nur im Kontext ostdeutscher Bedingungen, sondern als Ergebnis gesamtdeutscher Entwicklungen betrachtet. PEGIDA ist in dieser Sichtweise ein Resultat einer von Abstiegsängsten und Postdemokratisierungsprozessen geprägten Gesellschaft, in der die politische Repräsentation in die Krise geraten ist und von normativen Unordnungen geprägt ist. In dieser Konstellation entstehen neue autoritäre Syndrome, die das Unbehagen mit der Gesellschaft in ressentimentgeladene Fremdprojektionen umwandeln.

\section{Abstract}

In this paper PEGIDA is considered as a product of all-German developments, which could not be reduced to particular conditions. In this perspective, PEGIDA is interpreted as a result of a fear of social downward mobility and the post-democracy, which caused a crisis of political representation and a crisis of the normative foundations of politics. In this constellation, a new authoritarian syndrome is developing, which converts the discomfort among the people into resentment.

Ganz normale Bürger, die sich sorgen; als solche wollen die PEGIDA-Anhänger gesehen werden. Das ist nicht ganz falsch, und genau das macht es so beängstigend. Denn PEGIDA ist keine originär rechtsextreme Bewegung, sondern das Produkt einer nervösen, von Abstiegsängsten und Ressentiments durchdrungenen Gesellschaft, in der die Affektkontrolle verwildert. Erst an zweiter Stelle, in indirekter Form, kommt der Rassismus. Offiziell grenzt sich PEGIDA immer wieder von rechtsextremen 
Positionen ab. Man betont den bürgerlichen Charakter der eigenen Positionen, der sich auf die westlichen Werte der Aufklärung, Demokratie, Freiheit, Selbstbestimmung und Rechtsstaatlichkeit bezieht. Noch im Dezember 2015 veröffentlichte der Organisationskreis ein Positionspapier mit neunzehn Punkten. Erneut gab man sich humanistisch und republikanisch: Man sprach sich für die Aufnahme von Kriegsflüchtlingen und ihre menschenwürdige Unterbringung aus. Die unverdienten und unproduktiven Migranten sollten jedoch schneller und effektiver abgeschoben werden können. Die meisten Punkte waren zudem insofern wenig überraschend, da man für fast jede dieser Äußerungen in den letzten zwei Dekaden einen halbwegs prominenten Politiker aus den Reihen der CDU/ CSU oder der SPD finden kann, der exakt diese Forderung mit großem Aplomb in der Öffentlichkeit vertreten hat.

PEGIDA ist ein Ausdruck einer Radikalisierung der (unteren) Mitte, eines regressiven Aufbegehrens gegen eine marktkonforme Demokratie, in der die Ökonomie zur sozialen Instanz geworden ist. Bei PEGIDA versammeln sich gewissermaßen rechte Wutbürger.

\section{PEGIDA ist kein rein ostdeutsches Phänomen, sondern Resultat unterschiedlicher Gelegenheitsstrukturen}

Der Erfolg von PEGIDA verwundert viele, denn den Deutschen oder auch den Dresdnern geht es doch - gemessen an den europäischen und internationalen Desastern - relativ gut. Daran ändert auch die Flüchtlingskrise nichts, zumal PEGIDA schon lange vor dem großen Zustrom von Bürgerkriegsflüchtlingen entstand.

Dass PEGIDA sich vor allem in Dresden etablierte, lag sicherlich auch an besonderen lokalen Umständen: in kaum einem Bundesland ist die politische Kultur so konservativ, sind die Bürger so entfremdet von der Politik, und gerade bei den Männern ist die Angst vor dem erneuten Abgehängtwerden, wie sie es in den Wendejahren erfahren haben, groß. PEGIDA ist insofern zwar ein ostdeutsches, sächsisches Phänomen, aber es ist Ausdruck eines gesamtdeutschen geistigen Klimas, einer schon länger gärenden neo-autoritären Strömung. Dass es in Teilen der Bevölkerung gärt, war bereits durch den erstaunlichen Erfolg der Bücher Thilo Sarrazins oder dem Aufschwung von schrillen, neurechten Hasardeuren wie Jürgen Elsässer klar. Was aber überraschte, war, dass sich die Ressentiments zu einer lokalen sozialen Bewegung mit bundesweiter Ausstrahlung verdich- 
ten ließen. Denn trotz des ostdeutschen Schwerpunkts ist PEGIDA ein Phänomen, das nur aus einer gesamtdeutschen Perspektive zu verstehen ist.

PEGIDA hat einige Vorläufer, nicht zuletzt auch in Westdeutschland. Zum einen sind es die verschiedenen lokalen »Pro-Bewegungen« (z.B. Pro-NRW). Diese gaben sich bereits als Bürgerbewegungen aus, vertraten aber im Grunde offen anti-islamische Ressentiments und verfügten über offene Übergänge zu rechtsextremen Verbänden. Nur wenige Woche vor dem Beginn von PEGIDA gab es mit »Hogesa«-Krawallen den größten rechtsextremen Mob auf der Straße seit den neunziger Jahren.

Aus der Sicht des Autors ist der Schwerpunkt PEGIDAs in Ostdeutschland auch auf die besonderen »politischen Gelegenheitsstrukturen« zurückzuführen. Hierzu zählen u.a. ein offener Zugang zum politischen Feld, die Erosion politischer Bindungen und gespaltete Eliten (vgl. Tarrow 1996). In Sachsen haben vor allem Ministerpräsident Stanislaw Tillich und die regierende CDU lange Zeit PEGIDA verharmlost und die Aufmärsche als legitime Proteste »besorgter Bürger« dargestellt. Ferner traf PEGIDA in Dresden nur auf eine schwache Gegenmobilisierung, die zwischenzeitlich sogar aufgegeben hatte. So hatte PEGIDA Gelegenheit über mehrere Wochen einen eigenen Kern aus Anhängern zu stabilisieren und eine gewisse Legitimität vor Ort zu erreichen. Aus der Bewegungsforschung ist ferner bekannt, dass Gegenmobilisierungen ein Faktor sind, um die Dynamik existierender Bewegungen nachhaltig zu schwächen (Meyer/Staggenborg 1996). Während dies in Dresden nicht der Fall war, stießen die Versuche PEGIDA in Westdeutschland zu etablieren - etwa in München oder Frankfurt - auf eine beständige Gegenmobilisierung, die die PEGIDA-Demonstrationen zahlenmäßig in der Regel übertrafen.

\section{PEGIDA ist ein Ausdruck normativer Unordnungen im politischen System}

PEGIDA ist ein Protestphänomen normativer Unordnungen und Ausdruck einer Krise der Repräsentation der Volksparteien (Michelsen/Walter 2013). Vor 50 Jahren analysierte Otto Kirchheimer den Aufstieg der, wie er es nannte, Allerweltsparteien (Kirchheimer 1965). Kirchheimers Diagnose resultierte aus dem Wandel der weltanschaulichen Massenintegrationsparteien, wie man sie aus der Vorkriegszeit kannte. Die sozialistischen, konfessionellen oder konservativen Parteien wurden durch den Parteien- 
wettbewerb domestiziert, die Verschmelzung von Interesse und Ideen, von Weltanschauung und Gesellschaftsentwurf, wurde zugunsten eines entideologisierten Pragmatismus aufgegeben. Man repräsentierte nicht mehr die eigene Klientel, vertrat nicht mehr die Interessen der Mitglieder, sondern fokussierte auf die Maximierung der Wählerstimmen. Der Konsens gegenüber den Angelegenheiten des Staates überwog nunmehr zwischen den Allerweltsparteien - und wuchs. Aus der heutigen Perspektive jedoch war sein Befund verfrüht, wenn man etwa die programmatischen Präferenzen der SPD in der Nachkriegszeit mit ihren heutigen Positionen vergleicht. Man könnte fast sagen, erst fünfzig Jahre später sind die Volksparteien wirkliche Allerweltsparteien, die vor allem um die Mitte buhlen, aber ihre alte Klientel, ihre alten Werte aufgegeben haben. Die alten normativen, oder nach der älteren Begrifflichkeit: weltanschaulichen, Differenzen haben einer Unordnung Platz gemacht. Die SPD hat den Arbeitsmarkt liberalisiert, zeitweise die Rente mit 67 eingeführt, die Christdemokraten die Familienpolitik modernisiert, die Wehrpflicht abgeschafft und die Energiewende eingeleitet. Von den alten Konflikten ist nicht mehr viel übriggeblieben. Der Konsens der Parteien ist demokratietheoretisch mit dem Mangel behaftet, dass es kaum noch wahrnehmbare, zugespitzte Alternativen gibt. Wer mit der aktuellen Politik unzufrieden ist, findet im Grunde keine Kanäle der Artikulation für seine Kritik. Die Unterscheidung von links und rechts verschwimmt in der Mitte zusehends.

Heute sind die alten Volksparteien zu Kartellparteien geworden, sie vertreten kaum noch die Interessen ihrer Mitglieder, sondern teilen sich die Macht mit den anderen Kartellparteien derart auf, indem sie ihre ganze Energie dafür aufwenden, konkurrierende Parteien - links, rechts und libertär - aus dem Parlament und vor allem den Regierungen heraus zu halten, um ihre eigene Position zu festigen (Mair 2013). Damit geben sie aber in der Regel aber die letzten Reste politischer Grundsätze auf. Für viele erscheinen die politischen Konflikte zwischen Konservativen und Sozialdemokraten bzw. den Volksparteien dann nur noch als Narzissmus der kleinen Differenz.

Politische Prozesse erscheinen derweil als so komplex, dass man sie kaum noch durchblickt, die Entscheidungen post-demokratisch, als in Hinterzimmern abgesprochen, die Interessen des »kleinen Mannes « nicht mehr repräsentierend. Am Ende dieses Zirkels steht jedoch der subjektive Souveränitätsverlust der Bürger gegenüber der Politik. Die Krise der Repräsentation ließ viele Bürger ratlos zurück, sie flüchteten sich in mit- 
unter aggressive Affekte. Eine häufige Folge ist die Pauschalkritik: Politik, Wirtschaft, Medien - sie alle gehören zu einem vermeintlichen Establishment. Dieses Gefühl verstärkte sich vor allem durch die Schuldenkrise und Flüchtlingskrise in Europa, als es gerade in der Mittelschicht - medial in voller Lautstärke orchestriert - Sorgen um die eigenen Besitzstände gab.

\section{PEGIDA ist ein Protest der Postdemokratie}

PEGIDA ist gewissermaßen die regressive Variante neuer politischer Proteste in den letzten Jahren. Trotz der zumeist großen politischen Unterschiede wiesen diese teilweise verblüffende Gemeinsamkeiten auf. Sie wandten sich alle in einer relativ pauschalisierenden Kritik gegen das Establishment und bezogen sich auf die Montagsdemonstrationen der Wendezeit, nicht zuletzt, indem man für sich in Anspruch nahm, »das Volk« zu repräsentieren. All diese Bewegungen verbindet ein tiefer anti-institutioneller Impuls der Selbstermächtigung; selbst bei den mittlerweile fast vergessenen Piraten war dies der Fall.

Der Wutbürger, wie man ihn etwa bei den Protesten gegen Stuttgart 21 traf, demonstrierte gegen ein aus seiner Sicht unnötiges, teures, die Natur und die Stadt zerstörendes Großprojekt; aber es war auch Protest für mehr Bürgerbeteiligung. Von seiner Natur her war der Wutbürger eher links, ökologisch und libertär, aber seine soziale Basis, die expert citizens, vertraten ihre Position häufig in einer apodiktischen Weise, die keinen Widerspruch duldete. Hinter seinen plebiszitären, basisdemokratischen Orientierungen lauerte schon damals bei einigen eine »autoritäre Versuchung«, die auf Effizienz und Expertentum setzt (vgl. Marg/Walter 2013).

Bei den Occupy-Protesten ging es hingegen noch eher hippiesk zu. Man protestierte in den Camps gleichermaßen gegen die ungerechte Verteilung als auch die politischen Asymmetrien unter dem Signum der »99 \%«. Demokratische und soziale Anliegen waren hier Ausgangspunkt für eine neuartige Protestform, die sich allerdings den traditionellen politischen Formen entzog. Occupy distanzierte sich von den etablierten politischen Akteuren vehement. Man wahrte große Distanz zu den Parteien, verstand sich als jenseits von links und rechts und war programmatisch jenseits des eigenen Repräsentationsanspruchs der »99\%« nicht festgelegt (Décieux/Nachtwey 2014).

Occupy verschwand von der politischen Bühne, es folgten die sogenannten »Montagsmahnwachen«. Diese entstanden in Folge des Ukraine- 
Konfliktes. Auf der einen Seite war in der neuen, vom Westen unterstützten Regierung nach dem Sturz von Präsident Janukovic die faschistische Partei Swoboda vertreten, auf der anderen Seite wurden nationalistische Separatisten von russischer Seite unterstützt, Wladimir Putin annektierte kurzerhand die Krim. Die Mahnwachen mobilisierten, wie bereits die Occupy-Bewegung, ihre Anhänger vor allem im Internet, den Parteien traute man ebenfalls nicht. Man stellte sich auch bewusst in die Tradition der Montagsdemonstrationen der Wendezeit, und zu Anfang gab man sich ebenfalls basisdemokratisch. Aber diese Protestbewegung war bereits von einer autoritären Strömung bewässert. Seltsame Esoteriker, bizarre Verschwörungstheoretiker und Querfrontparvenues tummelten sich ganz selbstverständlich zwischen ernsthaft Friedensbewegten. Es handelte sich bei den Montagsmahnwachen nicht um eine rechte Bewegung im engen Sinne, aber der Schwelbrand des Ressentiments und des Antisemitismus hatte sich ausgebreitet (vgl. Daphi et al. 2015).

PEGIDA entspringt nicht dem gleichen Dunstkreis, ist aber des gleichen Geistes Kind. Eine soziale Bewegung verbindet man gemeinhin mit der Arbeiter-, Frauen- oder Ökologiebewegung. Bei vielen handelt es sich um Bewegungen für Gerechtigkeit, Anerkennung und Identität. PEGIDA ist gewissermaßen eine postdemokratische und identitäre soziale Bewegung: man sorgt sich um die kulturelle Integration und die Werte einer aus den Fugen geratenen Welt. Aber dieser Konflikt ist keiner um die Anerkennung ethnischer Differenz, sondern um den Erhalt von Etabliertenvorrechten.

\section{Soweit bekannt, ist PEGIDA vor allem ein Phänomen der prekären, abstiegsbedrohten Mitte}

Die alte Bundesrepublik war noch vom, wie es Ulrich Beck genannt hat, kollektiven »Fahrstuhleffekt« geprägt. Unten, Mitte und Oben fuhren gemeinsam im Fahrstuhl nach oben, so dass die weiterhin bestehenden Ungleichheiten an Bedeutung verloren - weil es allen besser ging. Den Fahrstuhleffekt gibt es nicht mehr. Während die einen, die ohnehin Wohlhabende und Spitzenverdiener sind, weiter nach oben fahren, geht die Reise für die Mehrheit der Arbeitnehmer nach unten. Und dies trifft nicht nur die wachsende Anzahl von Armen, sondern bis zur Mitte der Gesellschaft. Die Mitte ist in den unteren Bereichen geschrumpft, und in der Folge haben sich Abstiegsängste ausgebreitet (Nachtwey 2016). Und gerade 
die Angst um den eigenen Status kann ein Treiber von negativen Affekten, regressiven Geschichtsbildern und Verschwörungstheorien sein.

Wer jetzt nun eigentlich bei PEGIDA mitläuft, das lässt sich nicht mit Gewissheit sagen. Die bisherigen Anstrengungen sozialwissenschaftlicher Untersuchungen stießen in Dresden an ihre Grenzen, da die meisten Anhänger sich verweigerten. Jene Teilnehmer, die Auskunft gaben, waren nicht mehr ganz jung, in der großen Mehrheit männlich, überdurchschnittlich gebildet ${ }^{2}$, und sozioökonomisch gehörten viele zur (unteren) Mittelschicht (vgl. u.a. Vorländer/Herold/Schäller 2016). Leider differenzierten die Studien nur sehr wenig im Bezug zum Erwerbsstatus. So weit man aus den verschiedenen Quellen schließen kann, ist PEGIDA tatsächlich die sich selbst als sozial und kulturell bedroht sehende Mittelschicht: Erwerbstätige aus den Zwischenzonen von Sicherheit und Unsicherheit, deren Leben besonders stark aus dem täglichen Kampf der Selbstbehauptung besteht und Selbständige3.

In Europa waren es in der Vergangenheit vor allem die Modernisierungsverlierer aus der alten Arbeiterklasse, die - allerdings nicht in der Mehrheit - anfällig für rechtspopulistische Positionen waren (Spier 2010). Jetzt scheint dieser Virus auf die Mitte übergesprungen zu sein.

In der Nachkriegsgeschichte war die Mitte ein Ort des Ausgleichs, der Affektkontrolle und der Integration. Aber gerade wenn die Mitte sich bedroht fühlt und fragmentiert, kann sie einen ihr eigenen Radikalismus entwickeln (Geiger 1930). Der Nationalsozialismus war für viele Beobachter auch das Resultat eines »Extremismus der Mitte« (Lipset 1960). Von solch einer Konstellation sind wir weit entfernt, und die Mitte ist nach wie vor ein Stabilitätsanker der Demokratie. Aber die Mitte fragmentiert. Das Ressentiment ist ihr nicht mehr fremd, Teile der Mitte sind von einem autoritären Syndrom befallen. Dies hat ökonomische, soziale und politische Ursachen.

Für viele Bürger erscheint die soziale Welt immer weniger lesbar, ja angstbereitend zu sein. Alle Diskurse und Dispositive der Gesellschaft sind auf Aufstieg ausgerichtet, aber aus der Gesellschaft des sozialen Aufstiegs der alten Bundesrepublik ist eine Abstiegsgesellschaft geworden. Man trampelt auf der Stelle. Man gibt sich dem Wettbewerb hin, bildet sich, arbeitet mehr und entgrenzter, verdichtet die Poren des Tages produktiver im Dienste der Leistung. Man verzichtet auf Ansprüche an das gute Leben, ist pflichtbewusst und verhält sich konformistisch - aber es geht nicht voran. Die eigene Sicherheit sehen viele nun zur Disposition gestellt, sei es durch die Eurokrise, die soziale Ungleichheit oder den Zu- 
zug von Migranten. Man fühlt sich alleingelassen und sehnt sich nach Orientierung. »Die Anrufung der Gemeinschaft dokumentiert traditionale Sehnsüchte in posttraditionaler Zeit.«, schrieb Georg Vobruba (1986: 221) schon vor vielen Jahren. Aber es trifft durchaus noch den Nerv der Gegenwart.

\section{Der neue Autoritarismus korrespondiert mit der marktkonformen Demokratie}

In der Postdemokratie, so die Diagnose von Colin Crouch (2008), sind die formalen Institutionen und Prozesse der Demokratie intakt, aber sie erodieren endogen, da die politischen Entscheidungen zunehmend den Interessen der Wirtschaft und der Lobbyisten folgen. In der marktkonformen Demokratie hat sich diese Logik radikalisiert. Hier wird die Demokratie der Wirtschaft untergeordnet, es gilt die vollständige Rationalität der ökonomischen Alternativlosigkeit. Die Märkte werden zur sozialen und politischen Kontrollinstanz, die keinen Widerspruch duldet, die unsichtbare Hand stellt sich für viele als Faust dar, der Kapitalismus ist autoritär geworden. Im Grunde ist der autoritäre Geist in die meisten Politikfelder eingezogen: Die Politik der Austerität ist nicht nur autoritär, weil sie keine ökonomischen Alternativen zum Sparen vorsieht, sondern weil sie alle Ausgaben unter den Vorbehalt der Effizienz stellt. Im Falle der Arbeitsmigration unterscheidet der Diskurs zwischen »nützlichen« Fachkräften aus dem Ausland und den unproduktiven Belastungen des Sozialsystems durch Flüchtlinge.

Die Angst vor dem Abstieg bringt zudem einen ganz eigenen Autoritarismus hervor. Die Abstiegsängste, das ständige Sich-Bewähren-Müssen im gesellschaftlichen Wettbewerb, die Statuskämpfe um Anrechte auf Wohlstand, die enttäuschten Erwartungen an Aufstieg und Sicherheit führen zu einer »Entnormativierung« und »Verwilderung« sozialer Konflikte (Honneth 2013). Die in der alten Bundesrepublik relativ erfolgreiche Regelung und Befriedung sozialer Konflikte gerät aus der Bahn. Aber dieser Zusammenhang entsteht nicht automatisch, er hängt von vielen Faktoren und Zufälligkeiten ab. Was man jedoch mit großer Wahrscheinlichkeit sagen kann: Entstehen in solchen Konstellationen keine sozialen und solidarischen Proteste und Gemeinschaften, steigt die Wahrscheinlichkeit von ressentimentgeladenen Konflikten. 


\section{Der Kern von PEGIDA ist ein neues autoritäres Syndrom}

Bei PEGIDA handelt es sich in diesem Sinne um einen Protest, der ein neues autoritäres Syndrom der Mittelklasse reflektiert. Dieses Syndrom resultiert aus der oben skizzierten nervösen Gesellschaft, in der alte Gewissheiten nicht mehr gelten und künftige Erwartungen fragil erscheinen. Die Gesellschaft ist gleichzeitig unordentlich, instabil und autoritär. Kein Wunder also, dass soziale Pathologien entstehen. Pointiert ließe sich sagen: Die Bevölkerung wurde so lange post-demokratisch regiert, bis sie schließlich selbst post-demokratisch wurde.

Die bedeutendste Studie zum Autoritarismus stammt von Theodor W. Adorno. Ausgehend von Studien über die Verbreitung antisemitischer Einstellungen, wollten er und die anderen Forscher eines größeren Teams die dafür notwendigen allgemeinen psychologischen Grundlagen in den Persönlichkeitsdispositionen der Menschen untersuchen. Die autoritäre Persönlichkeit war für sie der Schlüssel, der Menschen antidemokratisch, ressentimentgeladen und potenziell faschistisch werden ließ. Die autoritäre Charakterstruktur zeichnet sich durch eine Reihe zusammenhängender Merkmale aus, die sich zu einem Syndrom verknüpfen: u.a. Konventionalität, Unterwürfigkeit und Aggression, Machtdenken, Mangel an Empathie, Stereotypisierungen, Zynismus, eine Obsession bezüglich Sexualität und schließlich Projektivität (Adorno 1995). Gerade der Punkt der Konventionalität ist von Bedeutung, da Adorno und seine Mitautoren und Mitautorinnen diese als »starre Bindung an die konventionellen Werte des Mittelstandes« definieren (ebd.: 45). Zum Wesen der autoritären Persönlichkeit gehört, dass man seine Aggressionen nicht gegen die Autorität richtet, sondern auf andere projiziert: Man überträgt die eigenen, aber einem selbst als unakzeptabel erscheinenden Triebe auf andere, um diese dann verurteilen zu können. So wird etwa die Missachtung von Frauenrechten, wie in den 19 Punkten von PEGIDA, als Grund für die Kritik am Islam aufgeführt - ausgerechnet von jener Sorte Mensch, die zu Hause in der Regel an sehr traditionellen Rollenvorstellungen festhalten. Denn gleichzeitig gehört zu den 19 Punkten, dass man endlich Schluss mit einer so genannten »Genderisierung « machen soll.

Politik und Wirtschaft erscheinen immer komplizierter - Verschwörungstheorien sind da ein Mechanismus der Komplexitätsreduktion. Ressentiments und Stereotype haben auch eine Orientierungsfunktion, 
primitive Formeln können die Komplexität reduzieren. Deshalb entsteht andererseits auch das Phänomen, auf Fremdgruppen die eigenen Abstiegssorgen zu projizieren. Gerade in Ostdeutschland gibt es häufig stärker ausgeprägte Gefühle der Deprivation, des zu kurz gekommen Seins. Die Nachwendejahre, als viele ihre soziale Position einbüßten und bis heute nur wenige Aufstiegsperspektiven dazu gekommen sind, haben tiefe Spuren hinterlassen. Deshalb will man aber auch die eigenen (imaginierten) Vorrechte, den eigenen Lebensstil um so erbitterter verteidigen. Der eigene Konformismus schlägt daher um in die Abwertung all jener, die anders und vermeintlich unproduktive Mitesser in einem unter Stress stehenden Sozialsystem sind: Flüchtlinge, Migranten und Moslems.

Autoritäre Haltungen zeichnen sich dadurch aus, dass man sich offizieller traditioneller Ideen und Werte bedient, man gibt ihnen jedoch in Wirklichkeit eine andere Bedeutung. So kritisiert man die Demokratie, weil sie nicht hält was sie verspricht - ist aber bereit, sie gegen ein System »auszutauschen, das alle Ansprüche auf menschliche Würde und Gerechtigkeit preisgibt« (ebd.: 199). Bei PEGIDA schlägt die Unzufriedenheit mit den demokratischen Institutionen in eine Verachtung eben dieser um. Das ursprünglich linke Topos vom »Verblendungszusammenhang« kehrt bei PEGIDA im Jargon der Nationalsozialisten als »Lügenpresse« wieder. Die autoritäre Persönlichkeit kritisiert die Demokratie nicht dafür, dass ökonomische Ungleichheiten immer wieder in Widerspruch zur politischen Gleichheit geraten. Sie neigt eher dazu dazu, die Demokratie im Grunde abschaffen zu wollen und die »direkte Herrschaft derjenigen herbeiführen, die sie ohnehin für die Mächtigen halten« (ebd.: 221). Insofern ist es nur folgerichtig, wenn auf den Demonstrationen der Ruf nach der Hilfe des lupenreinen Demokraten Wladimir Putin erschallt. In der Masse strömen die Individuen im Wunsch nach »Identifizierung« und »affektiver Gemeinsamkeit« - in Anlehnung an eine Autorität, der man sich auch wider realistischen Wissens unterwerfen kann (Freud [1921] 1993). Für einige ist dieser Führer bizarrerweise eben Putin, aber die institutionalisierte Autorität ist heute etwas Abstraktes: das Primat der Wirtschaft.

Autoritäre Mentalitäten entstanden für Adorno und Co. nicht im luftleeren Raum. Sie sind Ergebnis der Sozialisation der Individuen, des politischen und ökonomischen Umfeldes, aber vor allem des geistigen Klimas der Zeit. Nicht zuletzt relevante Teile der Mittelklasse wenden sich von einer »egalitär-redistributiven« Gesellschaft ab (Blühdorn 2013: 158). 
Dies zeigt sich empirisch jüngst auch in umfangreichen Studien. In der gesamten Bevölkerung sogar etwas stärker, aber eben auch in der Mitte ist ein »marktkonformer Extremismus « entstanden, der in Verbindung mit unternehmerischen Selbstoptimierungsnormen Abwertungen anderer befördert. Vor allem bei jenen, die Angst um ihren Lebensstandard haben, tritt dieser Extremismus mit doppelt so hoher Wahrscheinlichkeit auf (Zick/Klein 2014). Gerade die Unterwerfung unter die vermeintliche ökonomische Alternativlosigkeit setze »autoritäre Aggressionen« frei (Decker et al. 2014).

In Politik und Medien werden zwar zumeist demokratische Werte vermittelt, zugleich aber eifrig viele Stereotype bedient. Dies schlägt sich auch in den oben zitierten Studien zu Rechtsextremismus und Fremdenfeindlichkeit nieder. Dort kommt man zwar in vielen Einzelaspekten zu unterschiedlichen Ergebnissen, aber in den Grundzügen zu den gleichen Befunden: Der manifeste Rechtsextremismus ist in den letzten Jahren zurückgegangen, die Ressentiments sind jedoch gestiegen. Vor allem Muslimen, Sinti, Roma und Asylbewerbern wird zunehmend mit Vorurteilen begegnet. Jenseits der realen Probleme, die bestimmte Teile des Islams in europäischen Gesellschaften bereiten, ist vor allem die Islamfeindschaft in der Regel das neue Gewand des Rassismus, der vor allem die vermeintliche kulturelle Überlegenheit der westlichen Kultur herausstellt. Dazu passt auch das hohe Maß an Konfabulation - es leben nur 6 Prozent Muslime in Deutschland, aber in der Bevölkerung schätzt man ihren Anteil auf 19 Prozent.

\section{Fazit:}

\section{PEGIDA stößt in die Lücke der Repräsentation}

»Wir sind das Volk«: diese Parole diente in den Wendejahren der Opposition in Ostdeutschland als Klammer für ihre demokratischen Forderungen. »Wir sind das Volk«, das ruft man jetzt auch bei PEGIDA. Man nimmt für sich in Anspruch, die Verkörperung einer Bürgerschaft zu sein, die nicht mehr gehört wird. Aber wer ist nun dieses Volk und wer sind seine Bürger? Schließlich handelt es sich entgegen der Selbstbeschreibung von PEGIDA gewiss nicht um den durchschnittlichen Bürger. Die Bürgerschaft wird gemeinhin zwar als ähnlich bezüglich ihrer Zugehörigkeit, ihrer sozialen Lage, ihren Rechten und Pflichten sowie Identitäten gedacht. Aber die Bürger, und wenn man so will: »das Volk« 
ist gleichzeitig äußerst heterogen. Bei PEGIDA und den verwandten Bewegungen entsteht eine neuartige, rechtspopulistische rebellierende Bürgerschaft, die jeweils einen Anspruch der Verkörperung des Volkes oder der Bürgerschaft für sich beansprucht. Es wird leider wenig nützen, die Anhänger von PEGIDA darüber aufzuklären, dass die Idee einer Identifikation mit einer nationalen Leitkultur analytisch nicht aufgeht. An PEGIDA und der AfD zeigt sich das Potential für das Aufkommen einer neuen »regressiven Massenbewegung« (Neumann 1978: 284), die die Krise der Repräsentation durch die Volksparteien ausdrückt.

\section{Anmerkungen}

1 Dieser Text ist eine überarbeitete Fassung von Nachtwey 2015.

2 Wobei man auch immer berücksichtigen muss, dass mit steigendem Bildungsgrad auch die Bereitschaft steigt, an Umfragen teilzunehmen und den eigenen Bildungsgrad preiszugeben.

3 Inwieweit die traditionelle Arbeiterklasse, das Dienstleistungsproletariat oder das abgehängte Prekariat sich zu PEGIDA zugehörig fühlten, ist kaum festzustellen. Für solche Deutungen gibt es noch weniger Belege als für die hier präsentierte These.

\section{LITERATUR}

Adorno, Theodor W. (1995): Studien zum autoritären Charakter, Frankfurt a.M.

Blühdorn, Ingolfur (2013): Simulative Demokratie. Neue Politik nach der postdemokratischen Wende, Berlin.

Crouch, Colin (2008): Postdemokratie, Frankfurt a.M.

Daphi, Priska/Kocyba, Piotr/Neuber, Michael/Rucht, Dieter/Roose, Jochen/Scholl, Franziska/Sommer, Moritz/Stuppert, Wolfgang/Teune, Simon/Zajak, Sabrina (2015): Protestforschung am Limit. Eine soziologische Annäherung an Pegida, Berlin.

Décieux, Fabienne/Nachtwey, Oliver (2014): Postdemokratie und Occupy, in: Forschungsjournal Soziale Bewegungen 27, S. 75-88. 
Decker, Oliver/Kiess, Johannes/Brähler, Elmar/Kompetenzzentrum für Rechtsextremismus- und Demokratieforschung (2014): Die stabilisierte Mitte. Rechtsextreme Einstellung in Deutschland 2014, Leipzig. Freud, Sigmund ([1921] 1993): Massenpsychologie und Ich-Analyse, Frankfurt a.M.

Geiger, Theodor (1930): Panik im Mittelstand, in: Die Arbeit. Zeitschrift für Gewerkschaftspolitik und Wirtschaftskunde 7, S. 637-654.

Honneth, Axel (2013): Verwilderung des sozialen Konflikts. Anerkennungskämpfe zu Beginn des 21. Jahrhunderts, in: Axel Honneth/ Ophelia Lindemann/Stephan Voswinkel (Hg.). Strukturwandel der Anerkennung. Paradoxien sozialer Integration in der Gegenwart, Frankfurt a.M., S. 17-39.

Kirchheimer, Otto (1965): Der Wandel des westeuropäischen Parteiensystems, in: Politische Vierteljahresschrift 6, S. 24-41.

Lipset, Seymour M. (1960): Political Man. The social Bases of Politics, New York.

Mair, Peter (2013): Ruling the Void? The Hollowing of Western Democracy, London.

Marg, Stine/Franz Walter (Hg.) (2013): Die neue Macht der Bürger. Was motiviert die Protestbewegungen?, Reinbek bei Hamburg.

Meyer, David S./Staggenborg, Suzanne (1996): Movements, Countermovements, and the Structure of Political Opportunity, in: American Journal of Sociology 101, S. 1628-1660.

Michelsen, Danny/Franz Walter (2013): Unpolitische Demokratie. Zur Krise der Repräsentation, Berlin.

Nachtwey, Oliver (2016): Die Abstiegsgesellschaft. Über das Aufbegehren in der regressiven Moderne, Berlin.

Nachtwey, Oliver (2015): Rechte Wutbürger. Pegida oder das autoritäre Syndrom, in: Blätter für deutsche und internationale Politik 6o, S. 8190.

Neumann, Franz L. (1978): Angst und Politik, in: ders./ Alfons Söllner (Hg.). Wirtschaft, Staat, Demokratie. Aufsätze 1930-1954, Frankfurt a.M., S. 424-459.

Spier, Tim (2010): Modernisierungsverlierer? Die Wählerschaft rechtspopulistischer Parteien in Westeuropa, Wiesbaden.

Tarrow, Sidney (1996): States and Opportunities: The Political Structuring of Social Movements, in: Doug McAdam/John D. McCarthy/ 
Mayer N. Zald (Hg.). Comparative Perspectives on Social Movements, Cambridge, S. 41-61.

Vobruba, Georg (1986): Die populistische Anrufung der Gemeinschaft, in: Helmut Dubiel (Hg.). Populismus und Aufklärung, Frankfurt a.M., S. 221-247.

Vorländer, Hans/Herold, Maik/Schäller, Steven (2016): PEGIDA. Entwicklung, Zusammensetzung und Deutung einer Empörungsbewegung, Wiesbaden.

Zick, Andreas/Klein, Anna (2014): Fragile Mitte - Feindselige Zustände. Rechtsextreme Einstellungen in Deutschland 2014, Bonn. 